\title{
Wavelength Conversion of QPSK and 16-QAM Coherent Signals in a CMOS Compatible Spiral Waveguide
}

Da Ros, Francesco; Porto da Silva, Edson; Zibar, Darko; Chu, Sai T.; Little, Brent E. ; Morandotti, Roberto; Galili, Michael; Moss, David J. ; Oxenløwe, Leif Katsuo

Published in:

Integrated Photonics Research, Silicon and Nanophotonics 2016

Publication date:

2016

Document Version

Publisher's PDF, also known as Version of record

Link back to DTU Orbit

Citation (APA):

Da Ros, F., Porto da Silva, E., Zibar, D., Chu, S. T., Little, B. E., Morandotti, R., Galili, M., Moss, D. J., \& Oxenløwe, L. K. (2016). Wavelength Conversion of QPSK and 16-QAM Coherent Signals in a CMOS

Compatible Spiral Waveguide. In Integrated Photonics Research, Silicon and Nanophotonics 2016 SPIE International Society for Optical Engineering.

\section{General rights}

Copyright and moral rights for the publications made accessible in the public portal are retained by the authors and/or other copyright owners and it is a condition of accessing publications that users recognise and abide by the legal requirements associated with these rights.

- Users may download and print one copy of any publication from the public portal for the purpose of private study or research.

- You may not further distribute the material or use it for any profit-making activity or commercial gain

- You may freely distribute the URL identifying the publication in the public portal 


\title{
Wavelength Conversion of QPSK and 16-QAM Coherent Signals in a CMOS Compatible Spiral Waveguide
}

\author{
Francesco Da Ros ${ }^{1}$, Edson Porto da Silva ${ }^{1}$, Darko Zibar ${ }^{1}$, Sai T. Chu ${ }^{2}$, Brent E. Little ${ }^{3}$, Roberto Morandotti ${ }^{4}$, \\ Michael Galili' ${ }^{1}$, David J. Moss ${ }^{5}$, and Leif K. Oxenløwe ${ }^{1}$ \\ ${ }^{1}$ DTU Fotonik, Technical University of Denmark, DTU, Kongens Lyngby, 2800, Denmark, DK \\ ${ }^{2}$ Department of Physics and Materials Science, City University of Hong Kong \\ ${ }^{3} X i$ 'an Institute of Optics and Precision Mechanics, CAS, Xi'an, China PRC \\ ${ }^{4}$ INSR - Énergie,Matériaux et Télécommunications, 1650 Blvd Lionel Boulet, Varennes (Québec), J3X1S2, Canada \\ ${ }^{5}$ Centre for Microphotonics, Swinburne University of Technology, Hawthorn, VIC 3122, Australia \\ fdro@fotonik.dtu.dk
}

\begin{abstract}
We characterize a wavelength converter based on a 50-cm long low-loss spiral Hydex waveguide. A $10-\mathrm{nm}$ FWM bandwidth is shown over which low OSNR penalty $(<0.5 \mathrm{~dB})$ wavelength conversion of QPSK and 16-QAM is reported.

OCIS codes: (190.4390) Nonlinear optics, integrated optics; (190.4380) Nonlinear optics, four-wave mixing; (060.1660) Fiber optics and optical communications, coherent communications.
\end{abstract}

\section{Introduction}

All-optical wavelength conversion is one of the most promising signal processing techniques for future optical wavelength division multiplexed (WDM) networks, as it enables a more effective and efficient wavelength management. Wavelength conversion would enable routing in the optical domain [1], and thus lower the wavelength blocking probability, as well as provide more advanced functionality such as path protection and Kerr nonlinearity mitigation through optical phase conjugation [2]. For wavelength converters to be applied to optical networks, however, modulation format independent operation needs to be ensured. Furthermore, as coherent transmission systems using quadrature phase-shift keying (QPSK) to 16-quadrature amplitude modulation (16-QAM) are already commercially deployed, the stringent requirements in terms of optical signal-to-noise ratio (OSNR) and low phase noise needs to be addressed. Significant progress has been made for wavelength converters based on four-wave mixing (FWM) in highly nonlinear fibers (HNLFs), with conversion of dual-polarization 64-QAM signals recently being demonstrated [3]. Nonlinear processing in HNLFs is, however, hindered by stimulated Brillouin scattering (SBS). Even though techniques such as fiber straining [4] or phase dithering in counter-phasing operations [3] have been shown to mitigate the impact of SBS, they come at the price of a significant increase in device complexity.

An integrated wavelength converter based on a compact waveguide device offers the potential benefits of reduced footprint, cost and increased scalability. Additionally, several integrated material platforms have the added benefit of higher SBS immunity. Previous reports of integrated wavelength converters for advanced modulation formats have shown conversion of QPSK [5-7], 16-QAM [8, 9], and up to 128-QAM orthogonal frequency division multiplexing (OFDM) [10] using silicon [5, 7-10] and silicon-germanium [6] waveguides. However, these materials are well known to be limited by nonlinear absorption at telecom wavelengths, and this has motivated the search for other nonlinear material platforms such as AlGaAs [11], silicon nitride [12] and high index doped glass [13-16] that exhibit a much higher nonlinear figure-of-merit (FOM) [13] in the telecom wavelength range.

Here, we extend our recent results of [16] by providing both a static (continuous wave, CW) and dynamic (modulated signal, QPSK and 16-QAM) characterization of a wavelength converter based on an integrated 50-cm long low-loss spiral high-index doped glass waveguide under CW pumping. The conversion efficiency (CE) is measured and we show a conversion bandwidth of 10-nm. Wavelength conversion of 32-Gbaud QPSK and 10Gbaud 16-QAM is further reported and the signal quality after conversion is investigated. Only a moderate pump power of $22 \mathrm{dBm}$ is required for achieving idler BER below the hard decision forward error correction (HD-FEC) threshold $\left(\mathrm{BER}=3.8 \times 10^{-3}\right.$ ) with an optical signal-to-noise ratio (OSNR) penalty below $0.5 \mathrm{~dB}$ over a 10 -nm bandwidth. Based on our measurement of FWM conversion bandwidth for a 30-dBm pump power, a BER below the HD-FEC threshold could be achieved over a bandwidth exceeding $30 \mathrm{~nm}$. This constitutes the first demonstration of wavelength conversion of continuous data signals in a high index doped glass waveguide, confirming its potential for nonlinear optical signal processing.

\section{Waveguide characterization}

A scanning electron microscope (SEM) image of the waveguide is shown in Fig. 1(a) prior to deposition of $\mathrm{SiO}_{2}$ over-cladding. The spiral waveguide has a cross section of $1.45 \mu \mathrm{m} \times 1.50 \mu \mathrm{m}$, surrounded by a silica cladding [13]. 
The final device has been pigtailed with fibers glued directly to the sample using V-grooves for alignment. The total insertion loss, including propagation loss, mode converter loss (1.2 dB) and pigtail splicing loss is $10.5 \mathrm{~dB}$. The group velocity dispersion is anomalous, $(8 \mathrm{ps} / \mathrm{nm} \cdot \mathrm{km}$ at $1550 \mathrm{~nm}$, inset of Fig. 2(b)) and the nonlinear parameter $\gamma$ is $220 \mathrm{~W}^{-1} \cdot \mathrm{km}^{-1}$ [13]. The lower nonlinear parameter compared to silicon is compensated for by the significantly lower propagation losses leading to a longer effective length, as well as the ability to use larger pump powers without any impact from nonlinear loss [15]. A first continuous-wave (CW) characterization of FWM in the 50-cm long spiral waveguide is shown in Fig. 1(b) and (c). The conversion efficiency (CE), defined as the power ratio between idler and signal at the waveguide output, is measured by injecting two co-polarized CW waves, a strong pump and a weak signal, into the waveguide and measuring the optical spectrum at the output with an optical spectrum analyzer (OSA) as shown in Fig. 2.

The CE versus input pump power is reported in Fig. 1(b) for a signal-pump spacing of $1 \mathrm{~nm}$. The deviation from linearity above 28-dBm pump power is believed to be due to heating effects in the glue based pigtails, since no such saturation was observed at much higher peak powers (and lower average power) in low repetition rate signals [15]. Fig. 1(c) instead shows the CE spectrum for 30-dBm of pump power reporting a 3-dB FWM bandwidth of $10 \mathrm{~nm}$.
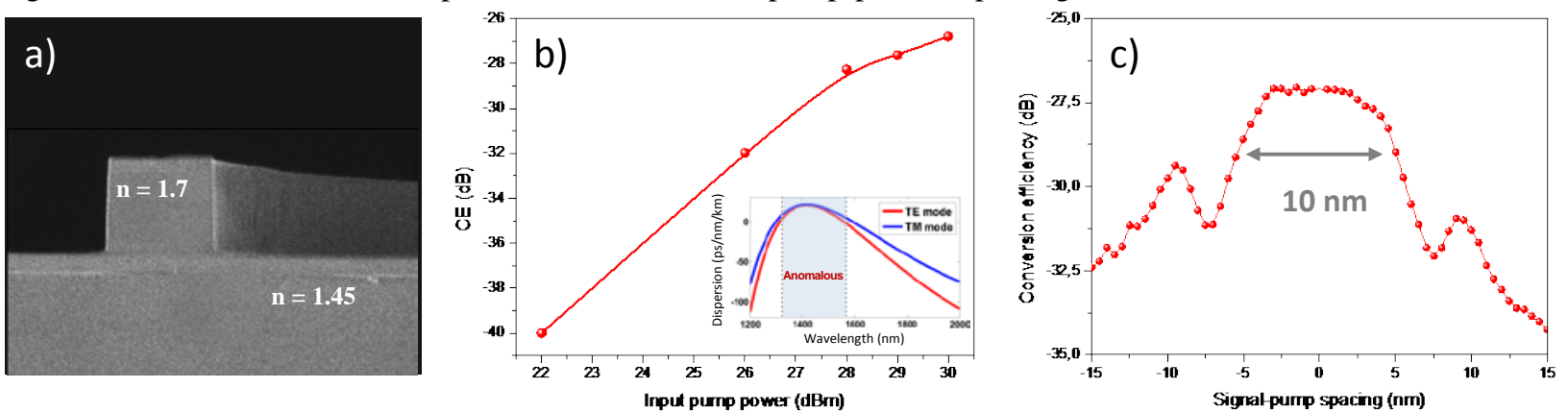

Fig.1. (a) SEM image of the waveguide prior to over-cladding deposition, (b) CE as a function of the input pump power (inset: estimated dispersion from [15]) and (c) CE bandwidth for a pump power of $30 \mathrm{dBm}$.

\section{Experimental setup}

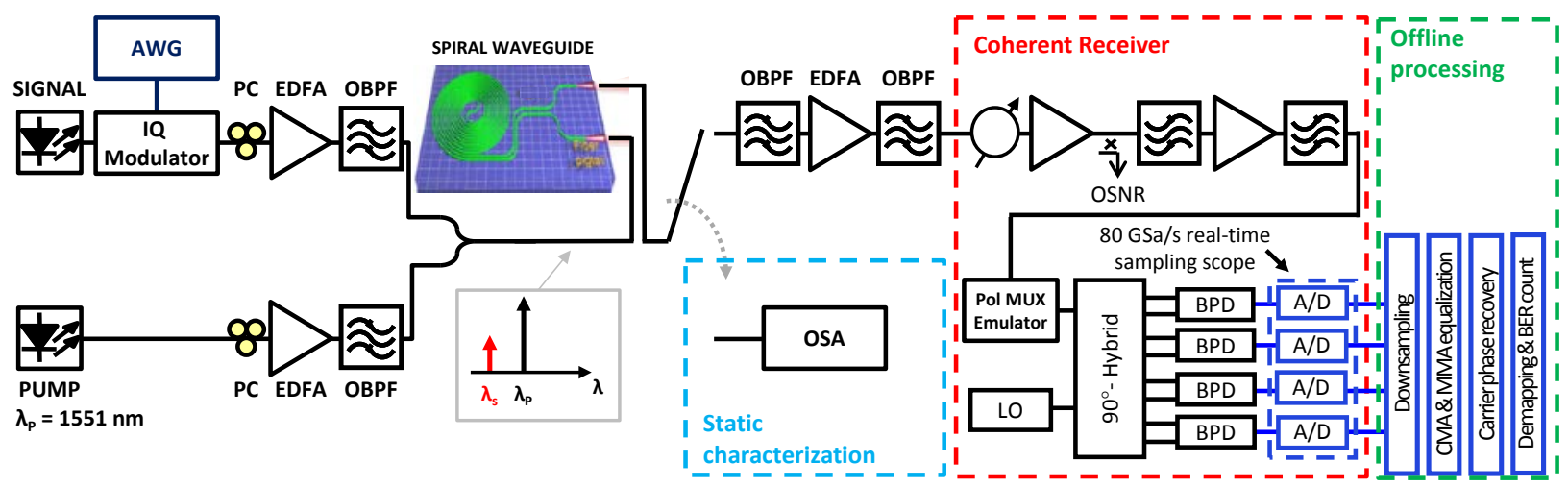

Fig.2. Experimental setup used for the static (CW) and dynamic (modulated signal) characterization of the $50 \mathrm{~cm}$ spiral waveguide.

The experimental setup is shown in Fig. 2. A QPSK or 16-QAM optical signal is generated by modulating a CW laser using a standard IQ modulator driven by a 64 Gsample/s arbitrary waveform generator (AWG) with 20 GHz of analog bandwidth. Another CW laser (linewidth $<100 \mathrm{kHz}$ ) at $1551 \mathrm{~nm}$ is used as a pump for the single-pump FWM scheme. The pump and signal are separately amplified in erbium-doped fiber amplifiers (EDFAs) and the amplified spontaneous emission noise (ASE) is filtered out by optical band-pass filters (OBPFs) with bandwidths of $0.8 \mathrm{~nm}$ and $0.5 \mathrm{~nm}$ for the signal and pump beams, respectively. The two waves are then coupled into the spiral waveguide with their polarizations aligned to maximize the conversion efficiency (CE).

The output of the waveguide is either sent to the OSA for the static characterizations shown in Fig. 1 or the idler is selected with a 2-nm wide BPF, pre-amplified and then filtered again with a 1-nm wide BPF to fully suppress pump and signal. The idler quality is then evaluated using a standard pre-amplified coherent receiver consisting of a noise loading stage, a pre-amplification stage, and a dual-polarization coherent receiver. A polarization emulator is used to generate a dual-polarization signal compatible with our receiver configuration. The received waveforms from the digital sampling oscilloscope (80 Gsamples/s, $33 \mathrm{GHz}$ ) are processed by offline digital signal processing 
(DSP) [17] including adaptive time-domain equalization using constant- and multi-modulus algorithms, (CMA) and (MMA), carrier recovery by decision-directed phase lock loop and bit error rate (BER) counting. The BER measurements are performed at moderate pump $(22 \mathrm{dBm})$ and signal $(16.5 \mathrm{dBm})$ powers since the idler OSNR was sufficient to achieve performance below the HD-FEC threshold. However, by increasing the pump power, based on the CE versus wavelength shown in Fig 1(c) and the quadratic scaling shown in [14], we estimate that a bandwidth exceeding $30 \mathrm{~nm}$ should be achievable.

\section{Results and discussion}

Fig. 3(a) shows the BER versus OSNR performance of the integrated wavelength converter for a 32-Gbaud QPSK signal as the signal-pump spacing is varied between $2 \mathrm{~nm}, 5 \mathrm{~nm}$ and $10 \mathrm{~nm}$. The idler BER is benchmarked against the back-to-back (b2b) signal measured directly at the output of the IQ modulator.

Varying the spacing from $2 \mathrm{~nm}$ to $10 \mathrm{~nm}$ causes a 3-dB reduction in received OSNR following the decrease in CE (Fig. 1(c)). Nevertheless all the idlers reach a BER below HD-FEC threshold with less than $0.5 \mathrm{~dB}$ of OSNR penalty.
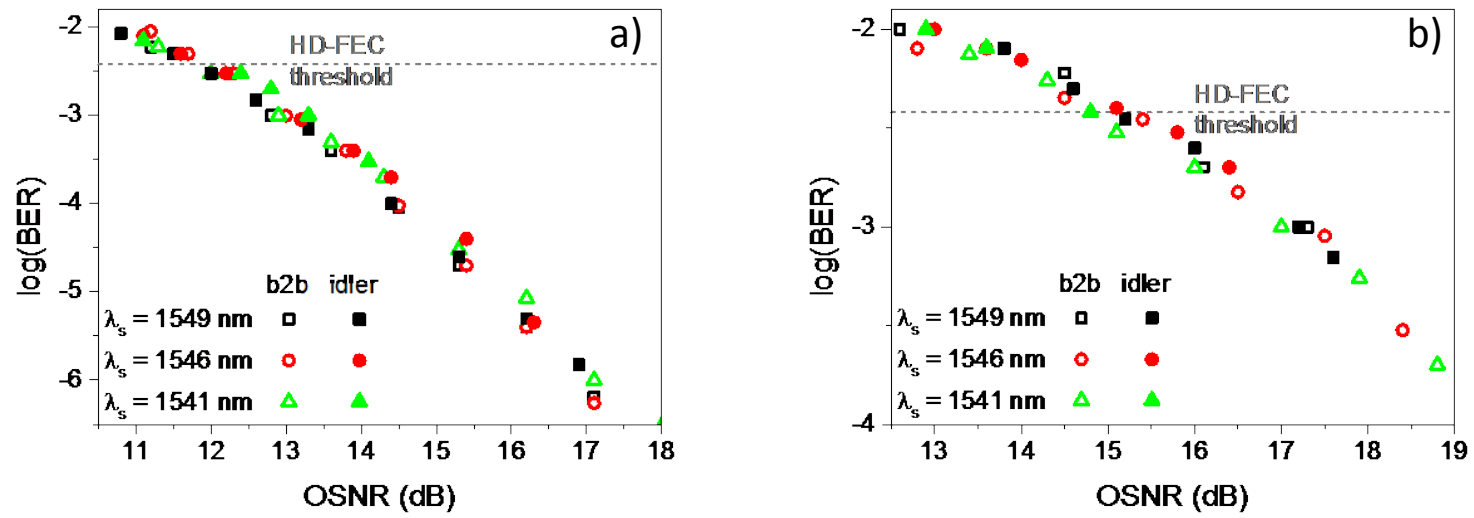

Fig. 3. BER performances as a function of the received OSNR (point A in Fig. 1) for (a) 32-Gbaud QPSK and (b) 10-Gbaud 16-QAM signal: comparison between back-to-back signal (open) and wavelength converted idler (full).

Fig. 3 (b) shows similar results for a 10-Gbaud 16-QAM signal with OSNR penalties still below $0.5 \mathrm{~dB}$ at the HD-FEC threshold. In this case the higher required OSNR for 16-QAM poses more stringent constraints allowing only a minimum BER slightly below the HD-FEC threshold for 10-nm signal-pump spacing. Higher pump powers or longer waveguides are expected to lead to a higher CE and thus a higher idler OSNR, as already discussed.

\section{Conclusion}

We characterize a wavelength converter based on a high index doped glass waveguide under CW pumping operation. The maximum CE and conversion bandwidth are measured for a 50-cm long spiral waveguide and wavelength conversion of 32-Gbaud QPSK and 10-Gbaud 16-QAM is demonstrated over a 10-nm bandwidth. The wavelength conversion allows achieving an idler BER below the HD-FEC threshold regardless of the moderate pump power with OSNR penalties below $0.5 \mathrm{~dB}$, compared to back-to-back.

\section{Acknowledgments}

This work was supported by the Center of Excellence Silicon Photonics for Optical Communications (SPOC) funded by the Danish National Research Foundation, by the National Science and Engineering Research Council in Canada, and by the Australian Research Council (ARC) discovery projects program.

\section{References}

[1] S. Yan, et al., J. Lightw. Technol. 33, 1586-1595 (2015).

[2] A. Yariv, et al., Opt. Lett. 4, 52-54 (1979).

[3] H. Nguyen Tan, et al. ECOC 2015, paper We.2.6.1.

[4] B.P.-P. Kuo, et al., Opt. Expr. 20, 18611-18619 (2012).

[5] D. Vukovic, et al., Opt. Expr. 23, 3640-3646 (2015).

[6] M.A. Ettabib, et al., Opt. Expr. 21, 16683-16689 (2013).

[7] X. Wang, et al., Opt. Lett. 39, 6122-6125 (2014).

[8] R. Adams, et al., Opt. Expr. 22, 4083-4090 (2014).

[9] X. Wang, et al., POEM 2015, paper OW2A.5.
[10] C. Li, et al., Opt. Lett. 39, 4583-4586 (2014).

[11] M. Pu, et al., OFC 2015, paper Th5A.3.

[12] C.J. Krückel, et al., Opt. Lett. 40, 875-878 (2015).

[13] D.J. Moss, et al., Nat. Phot. 7, 597-607 (2013).

[14] M. Ferrera, et al., Nat. Phot. 2, 737-740 (2008).

[15] D. Duchesne, et al., Opt. Expr. 17, 1865-1870 (2009).

[16] F. Da Ros, et al., OFC 2016, paper Tu2K.5.

[17] R. Borkowski, et al., IEICE Trans. Comm.. 97, 1528-1536 (2014). 